\title{
Crystal Growth of L-alanine with Glycine-based Oligopeptides: The Revelation for the Competitive Mechanism
}

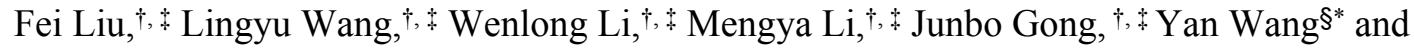 \\ Dandan Han ${ }^{\dagger}, *^{*}$ \\ ${ }^{\dagger}$ School of Chemical Engineering and Technology, State Key Laboratory of Chemical \\ Engineering, Tianjin University, Tianjin 300072, China \\ * The Co-Innovation Center of Chemistry and Chemical Engineering of Tianjin, \\ Tianjin 300072, China \\ $\S$ School of Pharmaceutical Sciences (Shandong Analysis and Testing Center), Qilu \\ University of Technology (Shandong Academy of Sciences), Jinan, 250014, PR China \\ *Email: wangyan57@tju.edu.cn (Yan Wang),Email: handandan@tju.edu.cn (Dandan Han)
}

The laser confocal micrographs of L-alanine crystals growing in aqueous solution with Gly and Gly-Gly-Gly are shown in Figure S1-S2. Compared with Figure 9, it can be found that there is no red fluorescent spot in L-alanine crystal growing in aqueous solution with Gly, which is because that Gly does not have fluorescent property so that the lattice incorporation of Gly can not be scanned by laser confocal scanning microscope. Besides, although Gly-Gly-Gly has fluorescent property, it can not embed into L-alanine due to its large steric hindrance. Therefore, in Figure S2, there is also no red fluorescent spot.

The effects of Gly and oligopeptides on the diffusion of L-alanine molecules around (1 20 ) and (l $\left.\begin{array}{lll}0 & 1 & 1\end{array}\right)$ faces were characterized through the molecule dynamics simulation, which show in Figure S3-S5. In these figures, the black solid line represents the diffusion with a ratio of 6 solutes(S) / 0 additive(A), the red dashed line represents the diffusion with $5 \mathrm{~S} / 1 \mathrm{~A}$ ratio, and the blue double dots dashed line represents the diffusion with a $3 \mathrm{~S} / 3 \mathrm{~A}$ ratio. Notably, there is almost no obvious change of mean square displacement of the L-alanine molecules diffusion around the (llll $\left.\begin{array}{ll}1 & 0\end{array}\right)$ and $\left(\begin{array}{lll}0 & 1 & 1\end{array}\right)$ faces with the change of concentrations of Gly and Gly-Gly. And as the concentration of Gly-GlyGly increases, the diffusion of L-alanine molecules around $\left(\begin{array}{lll}0 & 1 & 1\end{array}\right)$ face is not affected distinctly. Then, in the above cases, the diffusion effect cannot become the dominant mechanism to control crystal morphology and growth.

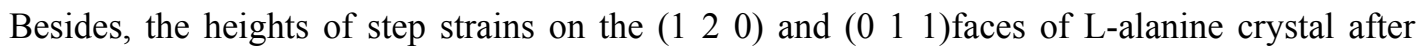
growing in aqueous solution with different concentrations of Gly and oligopeptides and pure water for $30 \mathrm{~min}$ are shown in Figure S6-S12, which are used to provide the specific data of step height change with the increasing concentrations of Gly and oligopeptides for the analysis of nanoscale 
surface structure of L-alanine crystal.
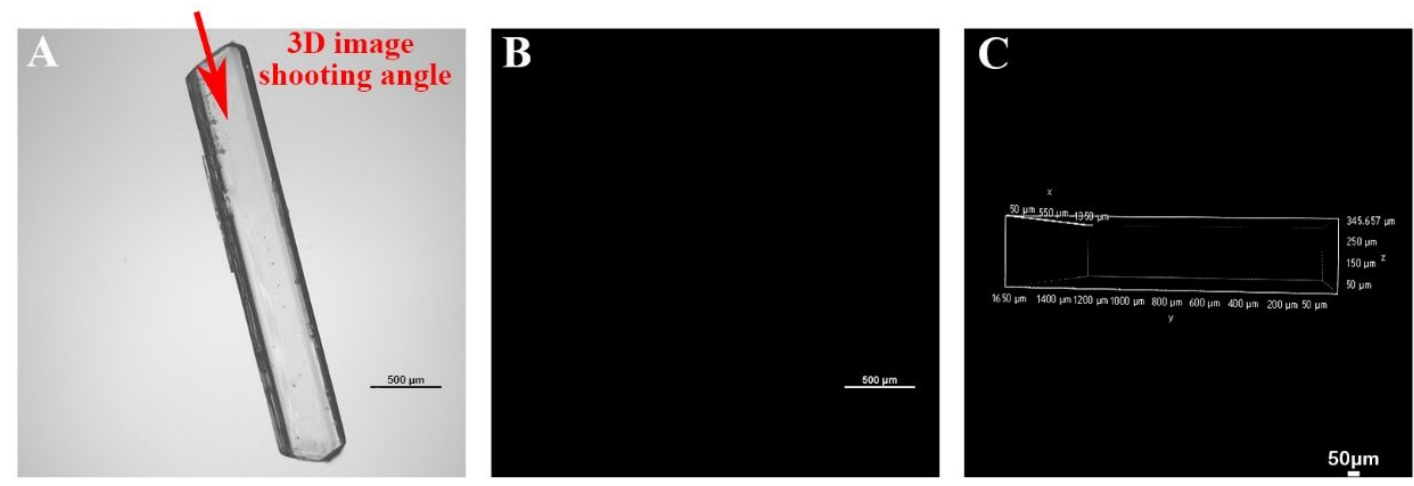

Figure S1. The laser confocal micrographs in (A) bright field, (B) 2D dark field, (C) 3D dark field of L-alanine crystals growing in aqueous solution with Gly
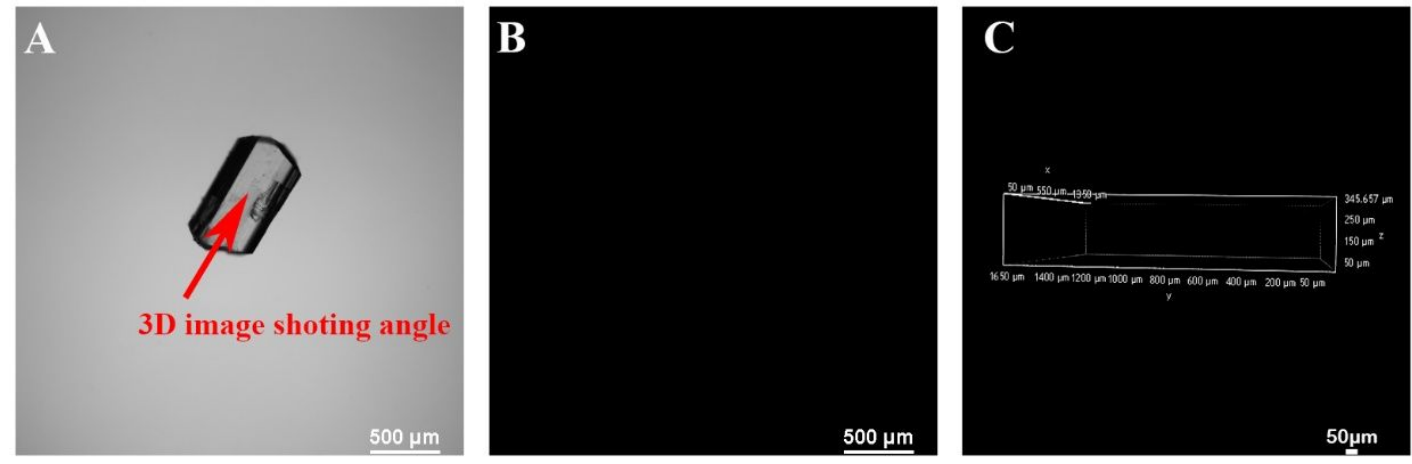

Figure S2. The laser confocal micrographs in (A) bright field, (B) 2D dark field, (C) 3D dark field of L-alanine crystals growing in aqueous solution with Gly-Gly-Gly
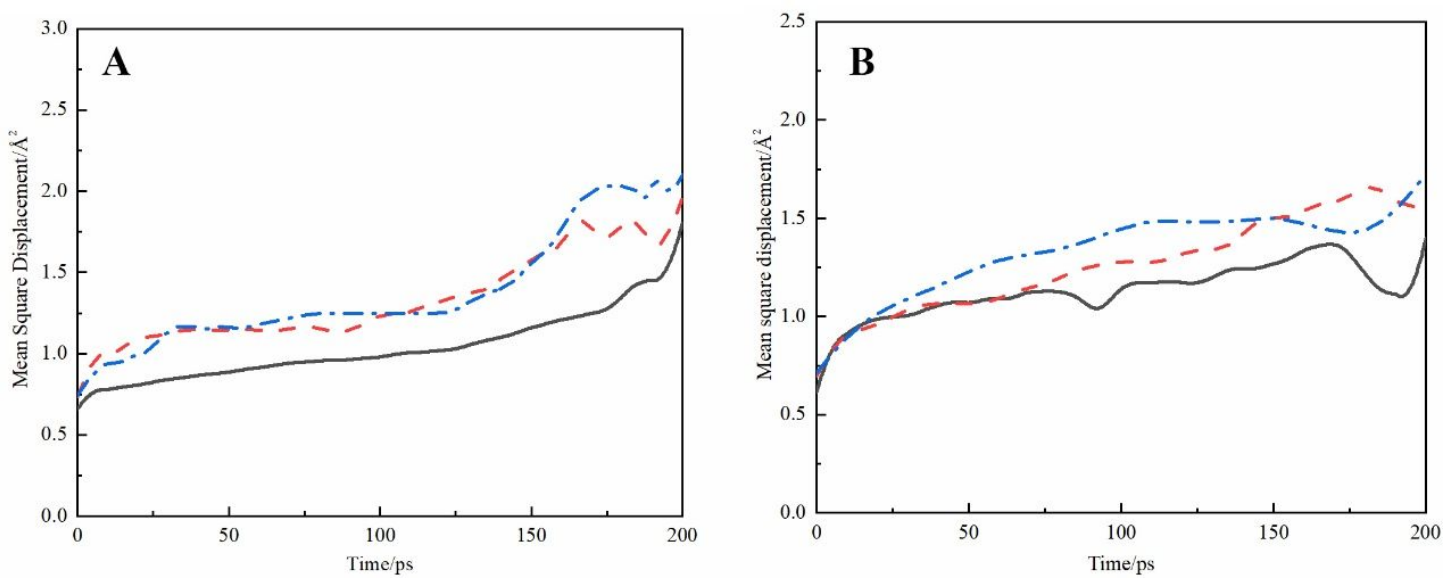

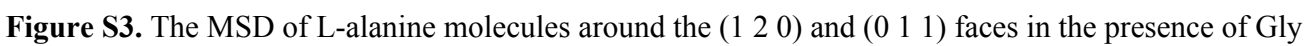



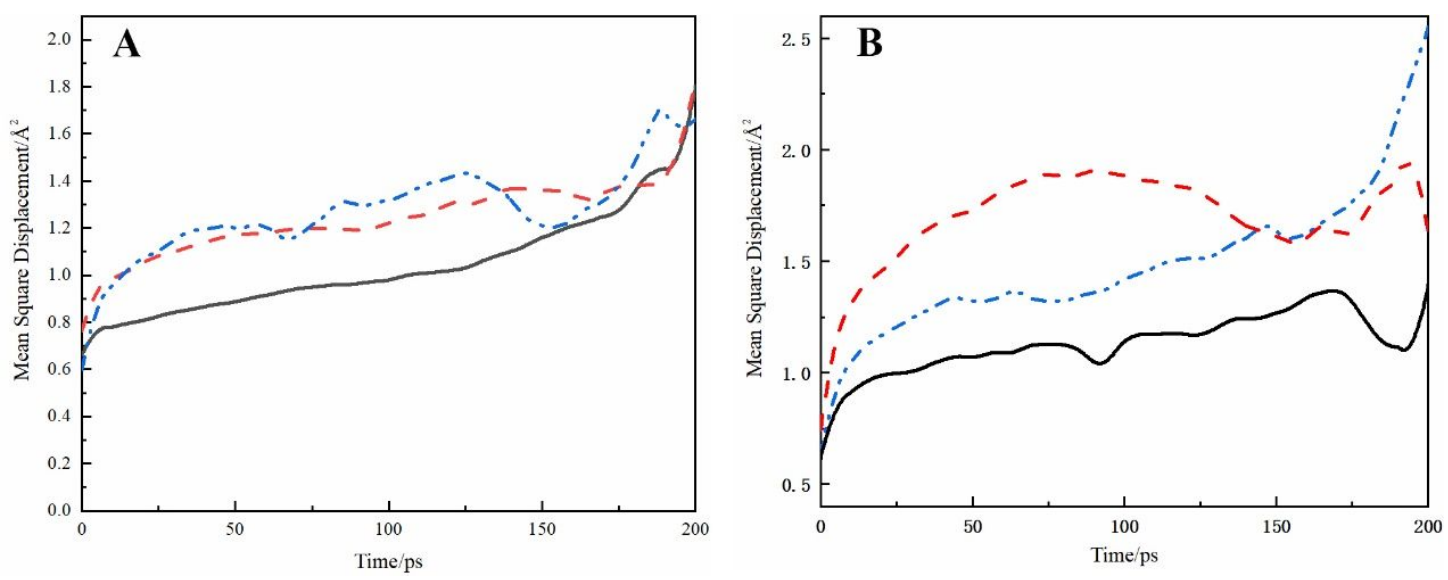

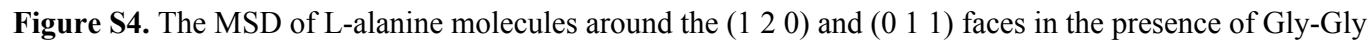

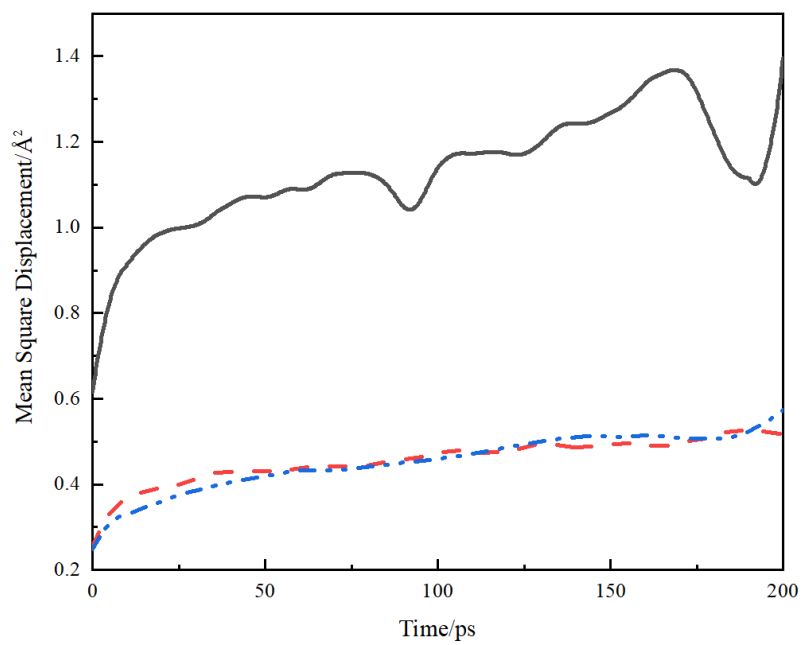

Figure S5. The MSD of L-alanine molecules around the $\left(\begin{array}{lll}0 & 1 & 1\end{array}\right)$ face in the presence of Gly-Gly-Gly 

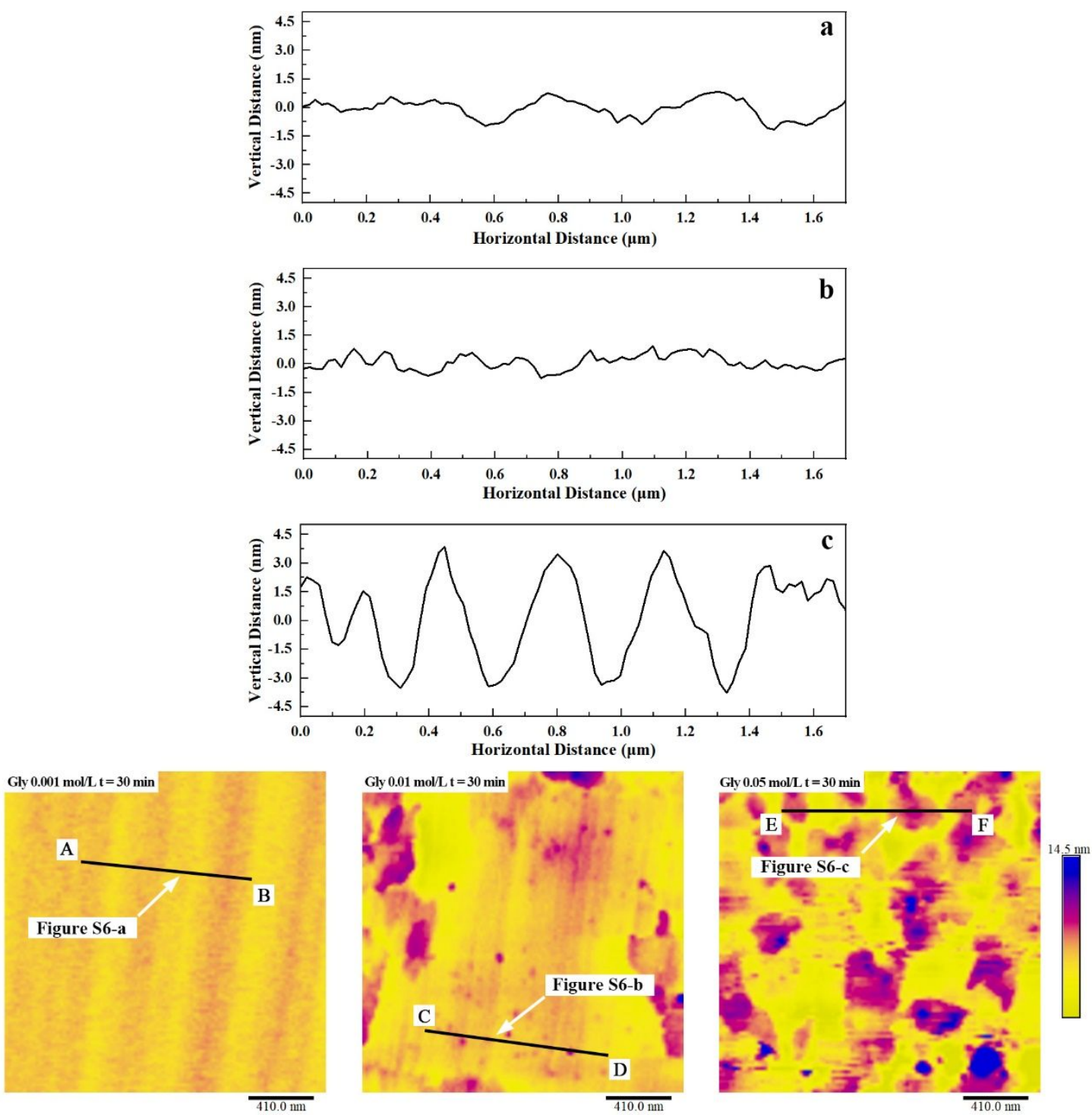

Figure S6. The step strains on the (1 20 ) face of L-alanine crystal after growing in aqueous solution with different concentrations of Gly for $30 \mathrm{~min}$ 

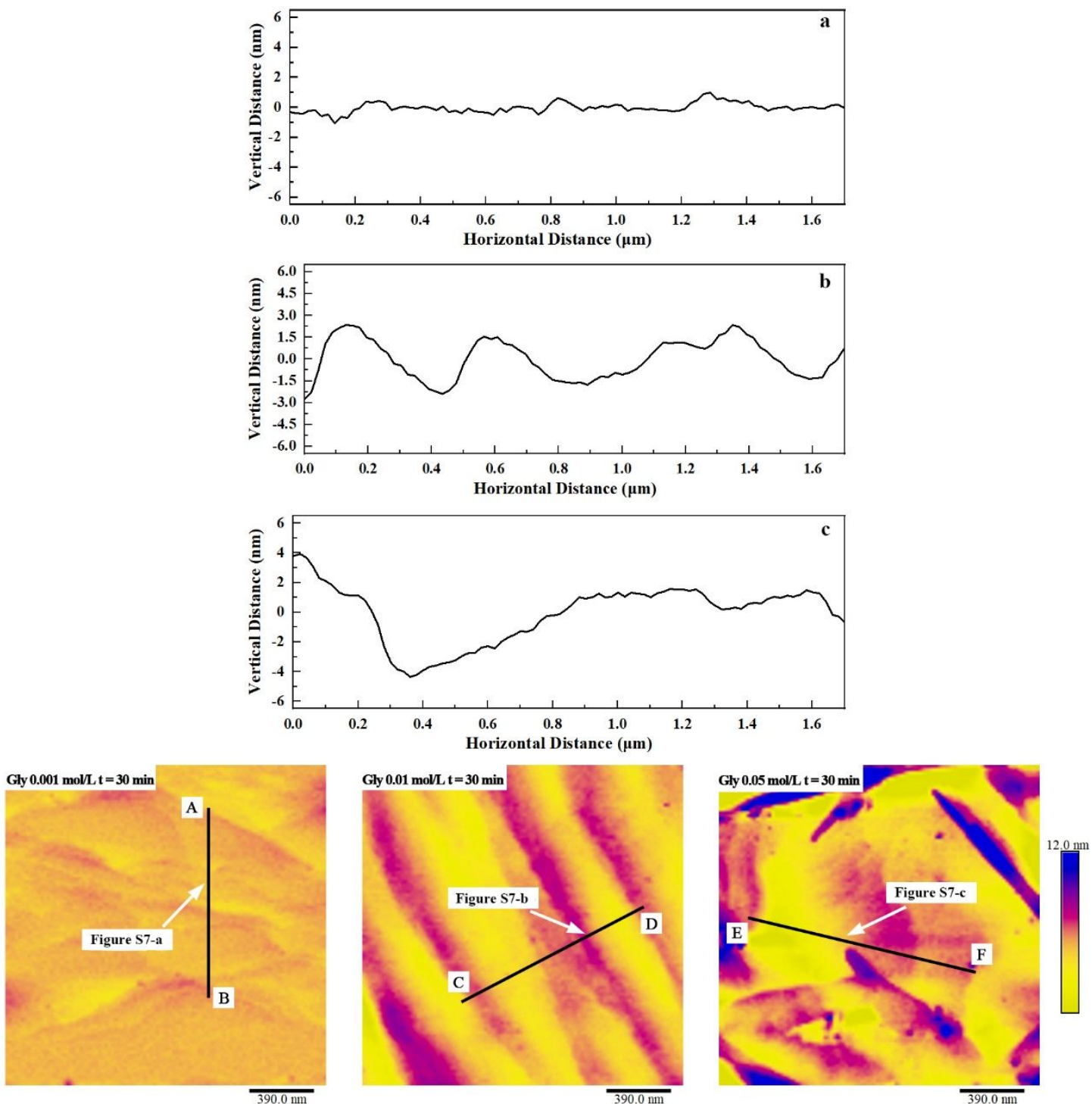

Figure S7. The step strains on the $\left(\begin{array}{lll}0 & 1 & 1\end{array}\right)$ face of L-alanine crystal after growing in aqueous solution with different concentrations of Gly for $30 \mathrm{~min}$ 

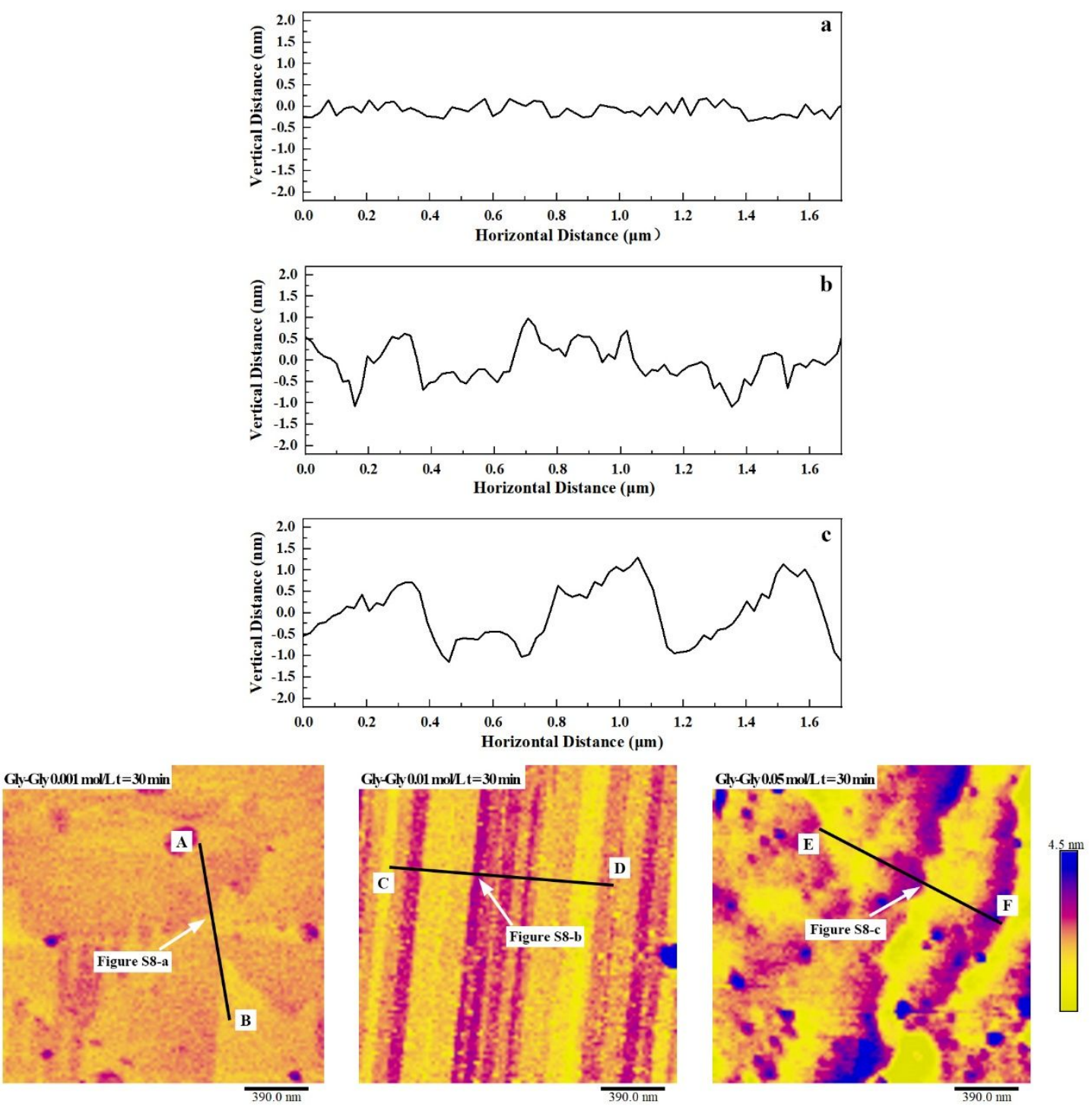

Figure S8. The step strains on the (1 20 ) face of L-alanine crystal after growing in aqueous solution with different concentrations of Gly-Gly for $30 \mathrm{~min}$ 

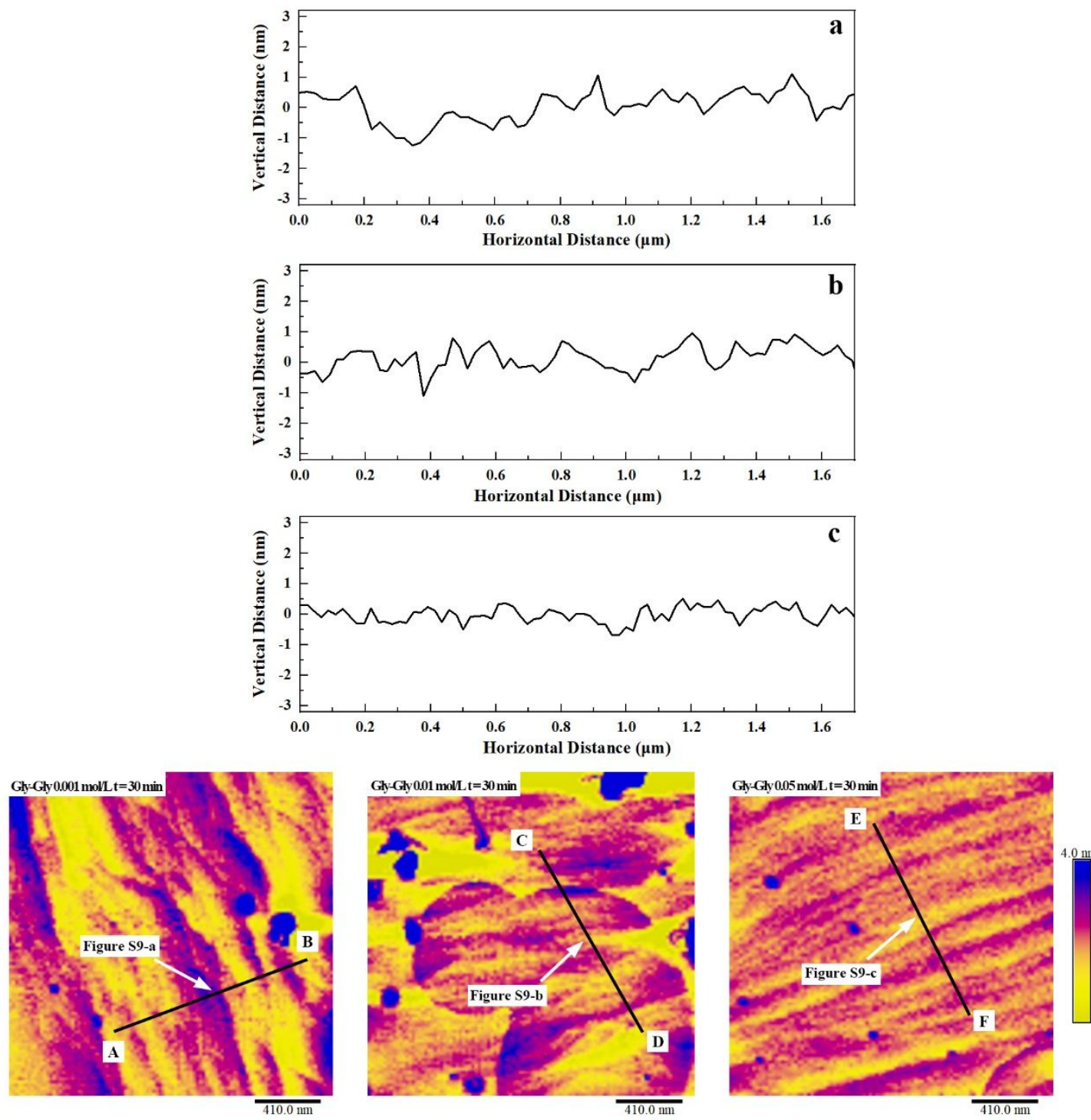

Figure S9. The step strains on the ( $\left.\begin{array}{lll}0 & 1 & 1\end{array}\right)$ face of L-alanine crystal after growing in aqueous solution with different concentrations of Gly-Gly for $30 \mathrm{~min}$ 

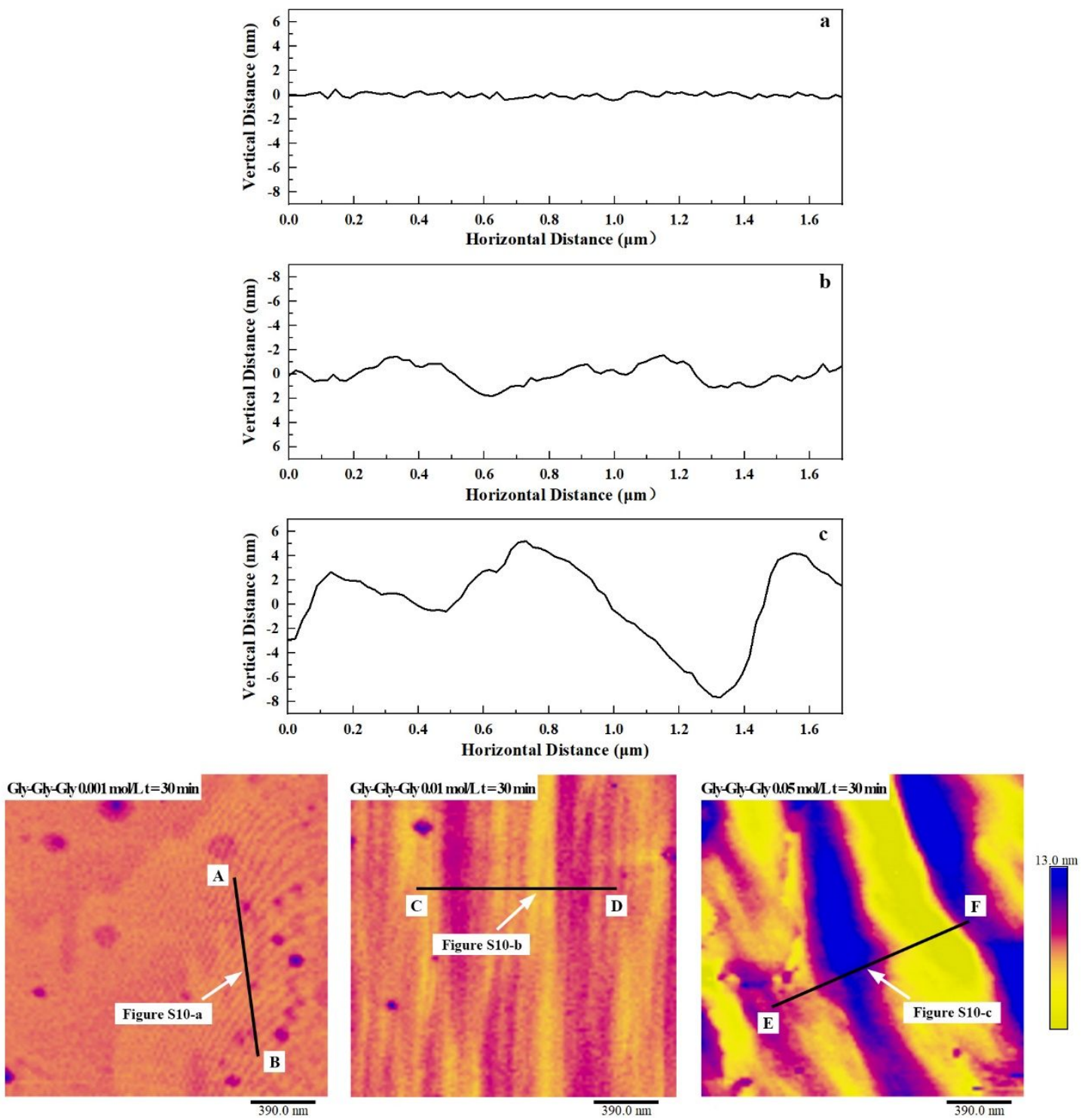

Figure S10. The step strains on the (1 20 ) face of L-alanine crystal after growing in aqueous solution with different concentrations of Gly-Gly-Gly for $30 \mathrm{~min}$ 

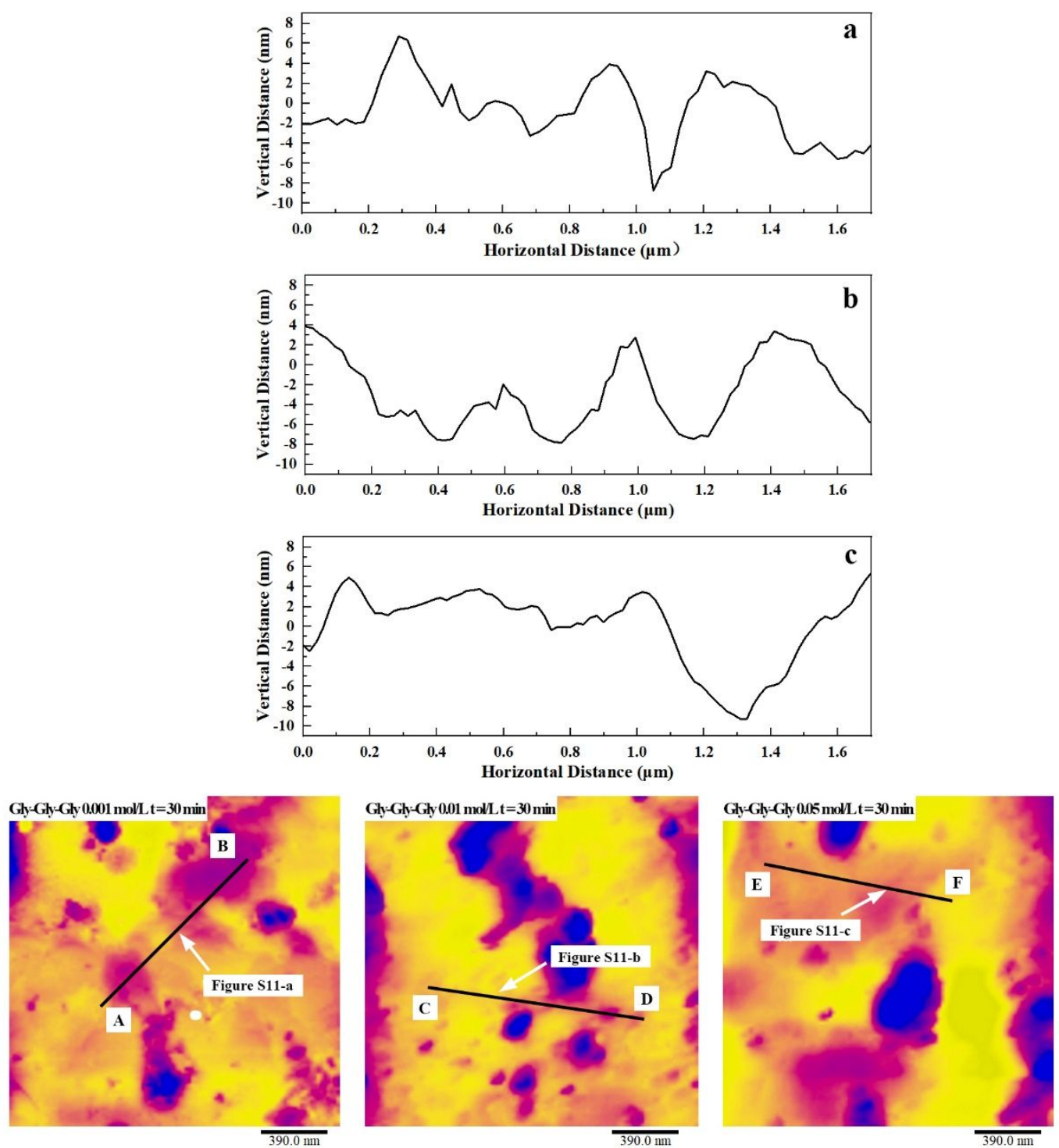

Figure S11. The step strains on the $\left(\begin{array}{lll}0 & 1 & 1\end{array}\right)$ face of L-alanine crystal after growing in aqueous solution with different concentrations of Gly-Gly-Gly for $30 \mathrm{~min}$ 

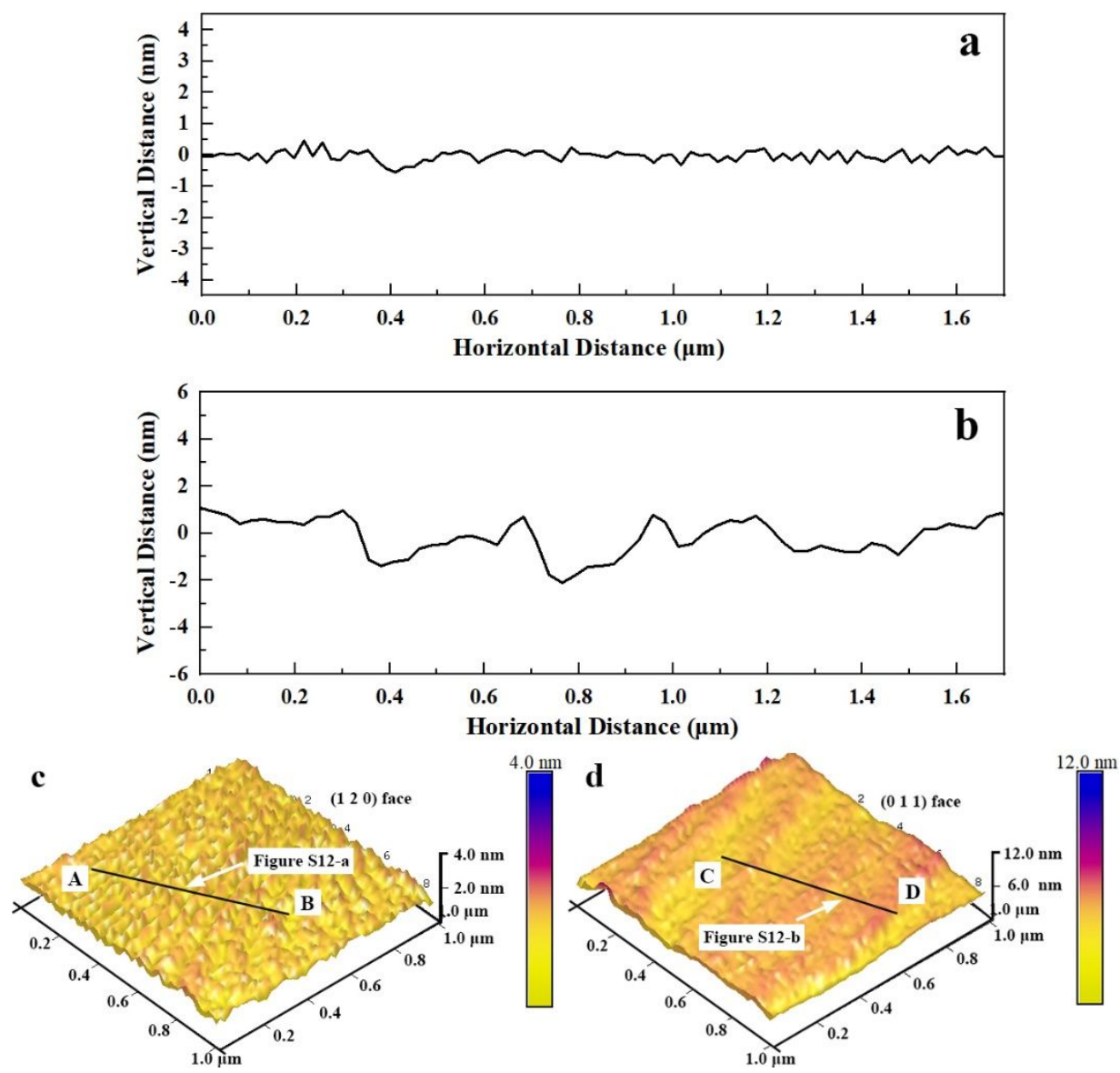

Figure S12. The step strains on ( $\left.\begin{array}{lll}1 & 2 & 0\end{array}\right)$ and $\left(\begin{array}{lll}0 & 1 & 1\end{array}\right)$ faces of L-alanine crystal after growing in pure water for $30 \mathrm{~min}$ 